\title{
Reno-protective effects of oral alkalizing agents in chronic kidney disease with aciduria: protocol for a randomized cohort study
}

Michiaki Abe ${ }^{1,2^{*}}$, Tetsuya Akaishi ${ }^{1}$, Mutsumi Shoji ${ }^{1}$, Takuhiro Yamaguchi ${ }^{3}$, Takashi Miki ${ }^{4}$, Fumitoshi Satoh ${ }^{2}$, Shin Takayama', Satomi Yamasaki ${ }^{5}$, Kazuhiko Kawaguchi ${ }^{5}$, Hiroshi Sato², Tadashi Ishii', Sadayoshi Ito ${ }^{2}$ and CKOALA study group

\begin{abstract}
Background: Aciduria caused by urinary excretion of acidic metabolic wastes produced in daily life is known to be augmented in patients with chronic kidney disease (CKD). To evaluate the reno-protective effect of oral alkalizing agents for the improvement of metabolic acidosis and neutralization of intratubular pH in the patients with mild stages of CKD. Also, to identify reno-protective surrogate markers in the serum and urine that can closely associate the effect of urine alkalization.

Methods: In this single-centered, open-labeled, randomized cohort study, patients with CKD stages G2, G3a and G3b, who visited and were treated at Tohoku University Hospital during the enrollment period were registered. We administered sodium bicarbonate or sodium-potassium citrate as the oral alkalinizing agents. A total of 150 patients with CKD will be randomly allocated into the following three groups: sodium bicarbonate, sodium-potassium citrate and standard therapy group without any alkalinizing agents. The data of performance status, venous blood test, spot urine test, venous blood-gas test, electrocardiogram, renal arterial ultrasonography and chest X-ray will be collected at 0, 6, 12 and 24 weeks (short-term study) from starting the interventions. These data will be also collected at 1 and 2 years (long-term study). The samples of plasma and serum and early-morning urine at every visit will be acquired for the analysis of renal function and surrogate uremic biomarkers.

The recruitment for this cohort study terminated in March, 2018, and the follow-up period for all the enrolled subjects will be terminated in December, 2020. The primary endpoint will be the development of originally-defined significant renal dysfunction or the occurrence of any cerebrovascular disease in the short-term study. The secondary endpoint will be the same endpoints as in the long-term study, or the patients with significant changes in the suggested the surrogate biomarkers.

(Continued on next page)
\end{abstract}

\footnotetext{
* Correspondence: michiabe@med.tohoku.ac.jp

'Department of Education and Support for Regional Medicine, Tohoku University Hospital, Sendai, Miyagi, Japan

${ }^{2}$ Division of Nephrology, Endocrinology and Vascular Medicine, Tohoku

University Graduate School of Medicine, Sendai, Miyagi, Japan

Full list of author information is available at the end of the article
}

(c) The Author(s). 2020 Open Access This article is licensed under a Creative Commons Attribution 4.0 International License, which permits use, sharing, adaptation, distribution and reproduction in any medium or format, as long as you give appropriate credit to the original author(s) and the source, provide a link to the Creative Commons licence, and indicate if changes were made. The images or other third party material in this article are included in the article's Creative Commons licence, unless indicated otherwise in a credit line to the material. If material is not included in the article's Creative Commons licence and your intended use is not permitted by statutory regulation or exceeds the permitted use, you will need to obtain permission directly from the copyright holder. To view a copy of this licence, visit http://creativecommons.org/licenses/by/4.0/ The Creative Commons Public Domain Dedication waiver (http://creativecommons.org/publicdomain/zero/1.0/) applies to the data made available in this article, unless otherwise stated in a credit line to the data. 
(Continued from previous page)

Discussion: The findings of this study will address the importance of taking oral alkalizing agents in the patients with early stages of CKD, furthermore they could address any new surrogate biomarkers that can be useful from early stage CKD.

Trial registration: Registered Report Identifier: UMIN000010059 and jRCT021180043.

The trial registration number; 150.

Date of registration; 2013/02/26.

Keywords: Chronic kidney disease, Oral alkalizing agents, Bicarbonate, Citrate, Single-centered and randomized cohort study

\section{Background}

Chronic kidney disease (CKD) is a chronic progressive disease related to eating habits and lifestyle [1-3]. These days, the increment of end-stage kidney disease (ESKD) is one of the biggest problems in the management of CKD patients [4]. If the patients with CKD are not properly treated, the disease may lead to cardiovascular events as well as ESKD and renal death $[5,6]$. The treatment to ameliorate tubulointerstitial dysfunction in the progression of CKD is important for preventing the final common pathway leading to ESKD [7, 8], which is yet to be verified by clinical research.

Generally, renal dysfunction may lead to metabolic acidosis and hyperkalemia, both derived from the accumulation of acidic metabolic wastes produced in daily life [9-11]. The acidic metabolic compounds, called uremic toxins (UTs), are the substances that primarily cause the aciduria [12, 13]. The accumulation of UTs is more common in CKD patients than in healthy individuals because of the disturbed urinary excretion from the damaged kidneys [14]. Some types of UTs are known to stimulate the ROS production, which may lead to further renal tubule-interstitial dysfunction $[15,16]$. Physiological acidification in renal tubules is not harmful itself; however, we previously reported that a stronger acid condition in renal tubules could result in an increased production of reactive oxidative stress (ROS), which could be aggravated by albuminuria [17]. In this previous study with a CKD animal model, an oral alkalinizing agent also improved aciduria by neutralizing renal intratubular $\mathrm{pH}$ and alleviating the latent metabolic acidosis.

In several past reports, treatments with some alkalinizing agents (sodium bicarbonate and sodium citrate) ameliorated renal damage in CKD patients with eGFR $<60$ $\mathrm{ml} / \mathrm{min} / 1.73 \mathrm{~m}^{2}$ [18-20]. In addition, intake of fruits and vegetables also ameliorated the renal dysfunction and high blood pressure [21]. Oral alkalinizing agents are also suggested to be useful for prolonging the initiation of hemodialysis by some unknown mechanisms, but this possible effect is still to be scientifically established.

In this protocol report, we planned a single-center randomly-allocated cohort trial, entitled as "Estimating the efficacy of the Oral ALkalinizing Agents in CKD; i.e. CKOALA study (UMIN000010059)", to verify the renoprotective effects of the oral alkalinizing agents in patients with mild stages of CKD, and also to seek renal protective surrogate markers, affected by the renotubular alkalization.

\section{Methods \\ Aims}

To verify the renal protective effects of oral alkalinizing agents by neutralizing the aciduria in the patients with mild stages of CKD.

\section{Objectives}

Our objectives are as follows:

1. To confirm the protective effects of oral alkalinizing agents on renal function in patients with mild and moderate-stages of CKD.

2. To compare the reno-protective effects of two types of oral alkalinizing agents, sodium/potassium citrate and sodium bicarbonate in CKD patients. Their effects will also be compared with that in the standard therapy group without oral alkalinizing agents.

3. To seek for the new surrogate markers in the serum and urine associated with early renal damage that can be cancelled by oral alkalinizing agents.

\section{Overview of study design}

This study is a single-center, open-label, randomlyallocated cohort study, with CKD patients in stages G2, G3a and G3b. To reduce the risk of sampling error, the enrolled patients will be randomly assigned based on the stratified random sampling process by using the random sequence generated by a computer software [22, 23]. The used variables for the stratification will be age (i.e. $\geq 65$, < 65 years old), sex, presence of diabetes mellitus, and eGFR (i.e. $\geq 46,<46 \mathrm{ml} / \mathrm{min} / 1.73 \mathrm{~m}^{2}$ ). With these four variables, the enrolled patients will be divided into 16 subgroups, and then randomly allocated into the below-described three cohort groups from each of the subgroups.

The enrolled patients will be allocated into the following three cohort groups: 1) sodium bicarbonate group, 2) sodium-potassium citrate group, and 3) standard 
therapy group without any alkalinizing agents. Then, these three cohort groups will be measured for the targeted biomarkers in the serum and urine in the "shortterm" study (i.e. at $0,6,12$ and 24 weeks after starting the intervention) and in the "long-term" study (i.e. at 1 and 2 years after starting the interventions) to assess the effects of oral alkalinizing agents. As for the urine samples, both early-morning urine and spot urine will be collected. The above-described overview of this cohort study is shown in Fig. 1.

\section{Collected variables}

At the time of registration, written informed consent is acquired from all the enrolled patients. At this time, the following information as to the patient's background are also collected: birthdate, sex, past medical history, complications, medication history, allergic history, smoking history, amount of alcohol intake, body weight and height.

The routine physical examinations, medication usage, and occurrence of adverse events are confirmed at every checkup in both the short-term and the long-term assessments.

Other data concerning the quality of life (QOL), laboratory test, chest X-ray, and physiological studies are confirmed at the set periods shown in Table 1. In detail, cardiac-thoracic ratio and presence of congestion for chest XRs, severe arrhythmia, angina pectoris and acute myocardial infarction for ECG and intrarenal blood flow and renal size for renal US. The QOL was assessed by using the SF- $8^{\text {ti }}$ Health Survey (Standard, Japanese version) by QualityMetric Incorporation and Shunichi Fukuhara (iHope international) [24]. The details of each blood test category and urine test category are listed in Table 2.

\section{Eligibility criteria for participants}

The patients, 20 to 80 years of age with CKD stages G2, G3a and G3b who were treated at Tohoku University Hospital between March, 2014 and March, 2018, will be eligible for this study. As for the exclusion criteria, patients who were administered with any medications or drinks that could have possible alkalinizing effects, or patients with tolvaptan within 30 days prior to the registration will be excluded. In addition, patients with renal hypouricemia, hyperkalemia, diabetes insipidus, hypernatremia with unknown origins, morning urinary $\mathrm{pH}$ higher than 6.8, or serious complications of heart disease or liver disease will also be excluded. Patients with hyperuricemia, morning aciduria, or metabolic acidosis will not be excluded.

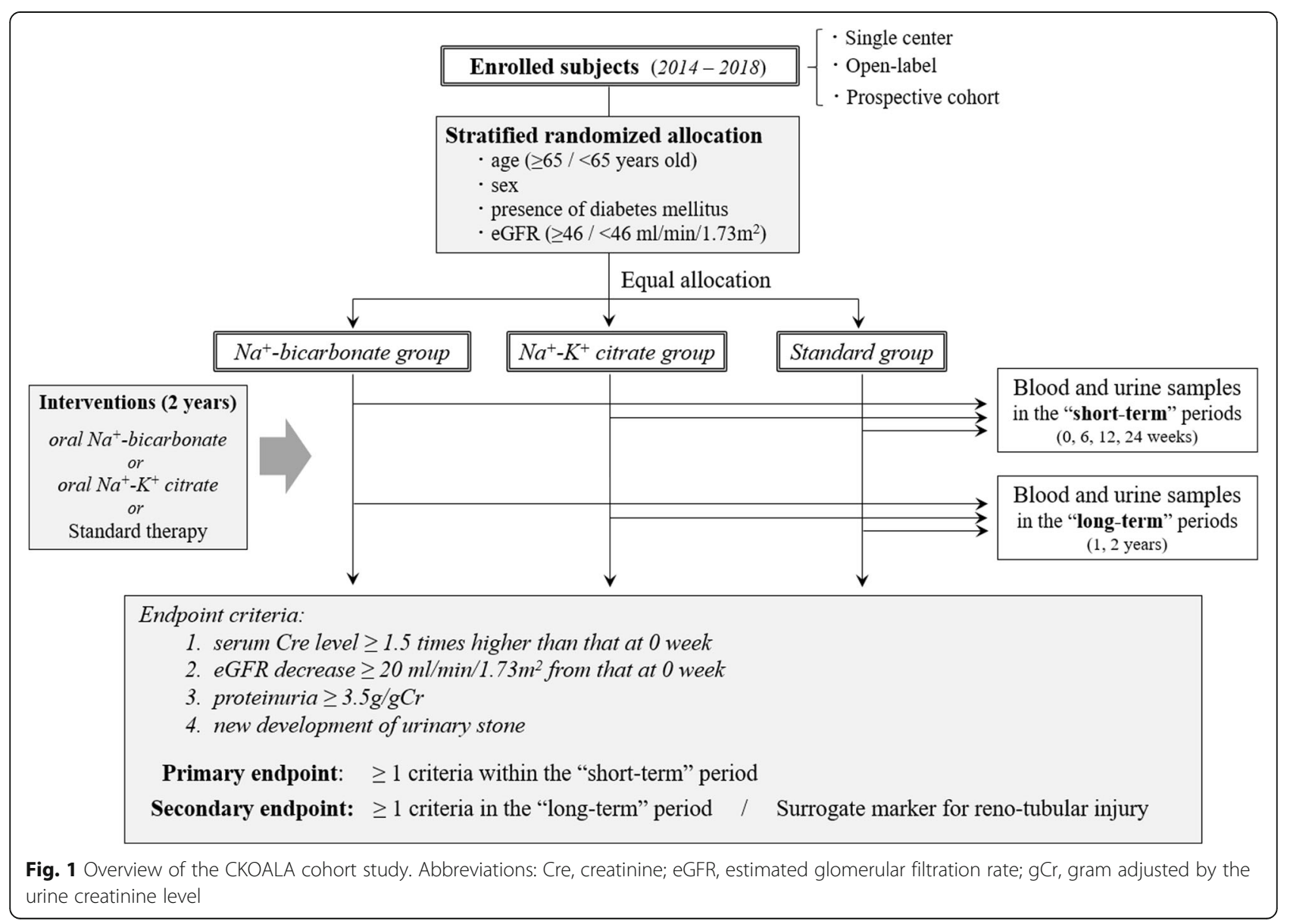


Table 1 Items and timing of laboratory and physiological examinations for short-term and long-term assessments

\begin{tabular}{|c|c|c|c|c|c|c|}
\hline & \multicolumn{4}{|c|}{ Short-term } & \multicolumn{2}{|c|}{$\underline{\text { Long-term }}$} \\
\hline & $0 w k$ & 6 wks & 12 wks & 24 wks & 1 year & 2 year \\
\hline $\mathrm{QOL}\left(\mathrm{SF}-8^{\mathrm{TM}}\right)$ & O & - & - & 0 & - & - \\
\hline \multicolumn{7}{|l|}{ Blood test } \\
\hline Category I & ० & ० & $\circ$ & $\circ$ & $\circ$ & ० \\
\hline Category II & ० & - & - & ० & $\Delta$ & $\Delta$ \\
\hline \multicolumn{7}{|l|}{ Urine test } \\
\hline Category I & ० & ० & $\circ$ & ० & ० & ० \\
\hline Category II & ० & ० & ० & $\circ$ & $\Delta$ & $\Delta$ \\
\hline ECG & ० & - & - & o & ० & ० \\
\hline CAVI, ABI & ० & - & - & ० & ० & ० \\
\hline Renal US & ० & - & - & $\circ$ & ० & o \\
\hline Chest X-ray & ० & - & - & ० & $\circ$ & $\circ$ \\
\hline
\end{tabular}

Details of the categories I and II of both blood and urine tests are listed in the following Table 2. The circles show that the examinations will be performed at each time without exceptions. The triangles show that the examinations will be performed if the measured values in the short-term period showed abnormality and their follow-up is suggested to be desirable

\section{Drug intervention}

Administration of each oral alkalizing agent, sodium/ potassium citrate or sodium bicarbonate, is started at $1.5 \mathrm{~g}$ per day. When early-morning urine $\mathrm{pH}$ is under $\mathrm{pH} 6.5$, the drug will be increased to $3.0 \mathrm{~g}$ per day. When early-morning urine $\mathrm{pH}$ is over $\mathrm{pH} 7.2$, the drug will be decreased or ceased up to less than pH 6.8.

Table 2 Details of categories I and II of both blood and urine tests

\footnotetext{
Category I (blood test)

Complete blood cell count, hemogram, hemoglobin, hematocrit, reticulocyte, Cre, eGFR, uric acid, $\mathrm{Na}^{+}, \mathrm{K}^{+}, \mathrm{Cl}^{-}$, lgG, $\mathrm{HCO}^{-}$, total cholesterol, $\mathrm{LDL}$-cholesterol, $\mathrm{HDL}$-cholesterol, triglyceride, glucose, $\mathrm{HbA1c}, \mathrm{CRP}, \mathrm{BUN}, \mathrm{Ca}^{2+}$, phosphate ion, magnesium ion, Fe, TIBC, UIBC, total protein, albumin, AST, ALT, LDH, ALP, transferrin, a1microglobulin
}

\section{Category II (blood test)}

Ferritin, whole PTH, plasma renin concentration, plasma aldosterone concentration, BNP, erythropoietin, bone specific alkaline phosphatase (BAP), insulin, cortisol, ACTH, leptin, adiponectin, endothelin-1 (ET-1)

\section{Category I (urine test)}

Creatinine, protein, albumin, $\mathrm{pH}, \mathrm{Na}, \mathrm{K}, \mathrm{Cl}$, urobilinogen, bilirubin, ketone body, occult blood, urinary sediments

\section{Category II (urine test)}

lgG, transferrin, retino-binding protein (RBP), a1-microglobulin, a2microglobulin, NAG, Neutrophil Gelatinase-Associated Lipocalin (NGAL), KIM-1, L-FABP, 8-isoprastan, 8-OHdG, type IV collagen, ET-1, angiotensinogen, MCP-1, thioredoxin, IL-1 $\beta$, IL-6, TNF- $\alpha$, aldosterone, $\mathrm{HCO}^{-}$, lactate, $\mathrm{pH}$

\section{Censoring criteria}

The censoring criteria as following; 1. hypernatremia, lower leg edema or early morning urine $\mathrm{pH} 7.2$ or higher for 2 months even after taking a drug holiday, 2. Serum potassium level keeps over $5.5 \mathrm{mEq} / \mathrm{L}$ or under $3.5 \mathrm{mEq} /$ L, 3. Adverse events such as exacerbation of symptoms on heart, liver or kidney, 4. subjects found to be ineligible after starting protocol treatment, 5 . subject who want to stop or withdrawal the consent, 6 . Subjects who do not visit because of moving etc. Data of censored subjects will be excluded.

\section{Endpoints}

The primary endpoints will be 1) the development of significant renal dysfunction, 2) the occurrence of any kind of cerebrovascular disease between 0 and 24 weeks (short-term study) from starting the interventions. The significant renal dysfunction will be defined as the conditions that fulfill at least one of the following four criteria; 1 . serum Cre level $\geq 1.5$ times higher than that at 0 week, 2. eGFR decrease $\geq 20 \mathrm{ml} / \mathrm{min} / 1.73 \mathrm{~m}^{2}$ from that at 0 week, 3 . proteinuria $\geq 3.5 \mathrm{~g} / \mathrm{gCr}, 4$. new development of urinary stones. The secondary endpoints will be the above-described criteria at 1 and 2 years (long-term study) from starting the interventions, and finding any kind of reno-protective marker associated with alkalinizing agents in both periods of study (Table 3).

\section{Sample size calculation}

A sample size was decided based on a previous study evaluating the reno-protective effect of the sodium bicarbonate [20], in which the incidence of ESKD under treatment with and without bicarbonate were 6.5 and $33 \%$, respectively. Based on this knowledge, we presumed the estimated incidence rate of ESKD in the sodium bicarbonate group and the sodium-potassium citrate group to be around 5 and 30\%, respectively. To achieve the significant level (i.e. type-I error rate) of $5 \%$ $(\alpha=0.05)$ and the statistical power of $80 \%(\beta=0.2)$ with the equally allocated three groups, we estimated the ideal sample size in each group to be 50 .

\section{Analysis}

The measured variables in the three groups will be compared by the analysis of variance (ANOVA), followed by the Bonferroni's post-hoc comparisons. If the variables show apparent non-normal distributions, Kruskal-Wallis test will be applied as a non-parametric test. Because of the simultaneous multiple comparisons, $p$-value $<0.01$ will be regarded as statistically significant. The chronological change of each variable in each group will be also evaluated. Values in each pair will be compared by the paired Student's t-test. If the variables show apparent non-normal distributions, Wilcoxon's rank sum test will 
Table 3 Primary and Secondary Endpoints

\begin{tabular}{|c|c|c|}
\hline Endpoints & Period & \\
\hline Primary & Short-term & $\begin{array}{l}\text { 1) The development of significant renal dysfunction } \\
\text { 1. serum Cre level } \geq 1.5 \text { times higher than that at } 0 \text { week } \\
\text { 2. eGFR decrease } \geq 20 \mathrm{ml} / \mathrm{min} / 1.73 \mathrm{~m}^{2} \text { from that at } 0 \text { week } \\
\text { 3. proteinuria } \geq 3.5 \mathrm{~g} / \mathrm{gCr} \\
\text { 4. new development of urinary stones } \\
\text { 2) The occurrence of any kind of CVD and the death }\end{array}$ \\
\hline \multirow[t]{2}{*}{ Secondary } & Lon-term & $\begin{array}{l}\text { 1) The development of significant renal dysfunction } \\
\text { 2) The occurrence of any kind of CVD and the death }\end{array}$ \\
\hline & Short-term \& Lon-term & $\begin{array}{l}\text { Reno-protective marker associated with alkalinizing agents } \\
\text { in both periods of study, for example, category II (blood } \\
\text { tests), categoryll (urine test), urine pH and unknown surrogate } \\
\text { biomarkers of early renal damage, including uremic toxins and } \\
\text { known metabolites measured by metabolomics. }\end{array}$ \\
\hline
\end{tabular}

be adopted. Comparisons of outcome with dichotomous data (i.e. frequency) between the groups will be performed by either of chi-squared test or Fisher's exact test, based on the achieved frequency in each cell. Lastly, to clarify the correlations between urine $\mathrm{pH}$, both for the early-morning urine and spot urine, and serum creatinine levels, Spearman's correlation coefficient for each pair will be calculated, followed by the test of no correlation.

\section{Discussion}

Recruitment to this cohort study terminated in March, 2018, and the follow-up period for all the enrolled subjects will be terminated in December, 2020.

We will seek for new therapeutic strategies using oral alkalinizing agents for the early stage CKD patients in this CKOALA study. Based on the previous study that showed the reno-protective effects of the oral sodium bicarbonate in the animal CKD model [17], we hypothesized that administration of oral alkalinizing agents for the patients with mild and moderate stages of CKD would delay the development of ESKD. This cohort study will confirm the reported benefits of oral alkalinizing agents for suppressing CKD progression in actual human cases.

In addition to the expectation that this study will confirm the therapeutic effects of oral alkalinizing agents for CKD patients, it would also clarify the relationship between the urine $\mathrm{pH}$ and the chronic renal damage. Because the oral alkalinizing agents have been suggested to only alkalize the urine without changing serum $\mathrm{pH}$, if the reno-protective effects of oral alkalinizing agents are confirmed, it will strongly suggest that aciduria is a definite risk factor to promote the renal damage in CKD patients.

One of the strengths of this study is that it will be conducted in a single center, which will enable us to exclude the risks of biases based on the differences between facilities and examiners. Meanwhile, there are some limitations. First, the number of enrolled subjects may remain low. If the results become negative due to inadequate sample size, we may extend the study period or plan additional randomized multi-center cohort study to achieve an adequate sample size. Another limitation is that this study will be an open-label trial. However, because the endpoints of this study are comprised of objectively measured serum and urine biomarkers that could not be affected by the preconceptions, biases based on the open-label style of this study to the given endpoints are not likely. Lastly, as for the patients' compliance to the medications, we will interview the intake habit at all the occasions of their hospital visits.

\section{Abbreviations}

CKD: Chronic kidney disease; ESRD: End-stage kidney disease; QOL: Quality of life; UT: Uremic toxins; ABI: Ankle brachial index; CAVI: Cardio ankle vascular index; ECG: Electrocardiogram; US: Ultrasonography; eGFR: Estimated glomerular filtration rate; LDL: Low-density lipoprotein; HDL: High-density lipoprotein; CRP: C-reactive protein; BUN: Blood urea nitrogen; TIBC: Total iron binding capacity; UIBC: Unsaturated iron binding capacity;

AST: Aspartate transaminase; ALT: Alanine transaminase; LDH: Lactate dehydrogenase; ALP: Alkaline phosphatase; PTH: Parathyroid hormone; BNP: B-type natriuretic peptide; BAP: Bone specific alkaline phosphatase; ACTH: Adrenocorticotropic hormone; ET-1: Endothelin-1; RBP: Retino-binding protein; NGAL: Neutrophil gelatinase-associated lipocalin; KIM-1: Kidney injury molecule 1; L-FABP: Liver-type fatty acid-binding protein; MCP-1: Monocyte chemotactic protein-1; IL-13: Interleukin-1 13; IL-6: Interleukin-6; TNF-a: Tumor necrosis factor-a; CKOALA: Estimating the efficacy of the oral alkalinizing agents in CKD

\section{Acknowledgements}

We appreciate the all member of CLOALA study group; Toshiki Nakai, Koichiro Nishioka and Satoshi Hashimoto (Nippon Chemiphar Co., Ltd) and Hideyasu Kiyomoto, Keisuke Nakayama, Masataka Kudo, Rei Morimoto, Takashi Nakamichi, Susumu Ogawa, Mariko Miyazaki, Shin Takayama and Takehiro Numata who helped the enrolment. We are thankfulness to Atsuko Masaura, Mayuki Endo, Kotoha Sasaki, Yoshiko Osada and Reiko Kokubun for the office work, and Dennis Nishimura for English proofreading.

\section{Authors' contributions}

MA did the initial idea for the study and making the protocol. TY, TM and TA contributed on the acquiring data and data analysis. SY and KK contributed technical advice to research assistants and procedure document for sample handing. MS, FS, ST, TI, HS and SI provided clinical registration. All authors have checked and approved the final manuscript.

\section{Funding}

This study is supported by the JSPS KAKENHI Grant-in-Aid for Scientific Research (C) (No; 16 K08487) and the cooperating research funding from 
Nippon Chemiphar Co., Ldt. The researchers of the funding company, SY and KK, contributed in the design of the study. For the details, technical advice to research assistants and procedure document for sample handing at the protocol designing.

\section{Availability of data and materials}

We informed the possibility that the samples and analysis results could be provided to other research institutes for secondary use, but personally identified information should not be used.

The data that support the findings of this study are available from the Data center of Clinical Research, Innovation and Education Center (CRIETO) of Tohoku University Hospital, but restrictions apply to the availability of these data, which were used under license for the current study, and so are not publicly available. Data are however available from the authors upon reasonable request and with permission of the Data center of CRIETO.

\section{Ethics approval and consent to participate}

All of the process of this study was approved by the Institutional Review Board of Tohoku University Hospital (IRB No; 2012-2-100-1). The study was performed in compliance with the Helsinki Declaration of 1964 and later versions.

The research doctors shall inform patients about the explanatory document and the consent forms are signed and dated by the subjects individually. The forms are stored. Every registration was carried out up to 0-week-visit day after obtaining the consent, and was allocated into the three groups.

\section{Consent for publication}

Not Applicable.

\section{Competing interests}

This study was designed and funded by Nippon Chemiphar Co., Ldt. The sponsor had no control over the interpretation, writing, or publication of this trial.

\section{Author details}

${ }^{1}$ Department of Education and Support for Regional Medicine, Tohoku University Hospital, Sendai, Miyagi, Japan. ²Division of Nephrology, Endocrinology and Vascular Medicine, Tohoku University Graduate School of Medicine, Sendai, Miyagi, Japan. ${ }^{3}$ Clinical Research, Innovation and Education Center, Tohoku University Hospital, Sendai, Miyagi, Japan. ${ }^{4}$ Clinical Physiology Center, Tohoku University Hospital, Sendai, Miyagi, Japan. ${ }^{5}$ Medical Affairs Department, Nippon Chemiphar Co., Ltd., Tokyo, Japan.

Received: 27 June 2019 Accepted: 15 April 2020

Published online: 22 April 2020

\section{References}

1. Rysz J, Franczyk B, Cialkowska-Rysz A, Gluba-Brzozka A. The Effect of Diet on the Survival of Patients with Chronic Kidney Disease. Nutrients. 2017;9:5.

2. Kelly JT, Palmer SC, Wai SN, Ruospo M, Carrero JJ, Campbell KL, et al. Healthy dietary patterns and risk of mortality and ESRD in CKD: a metaanalysis of cohort studies. Clin J Am Soc Nephrol. 2017;12(2):272-9.

3. Palmer SC, Maggo JK, Campbell KL, Craig JC, Johnson DW, Sutanto B, et al. Dietary interventions for adults with chronic kidney disease. Cochrane Database Syst Rev. 2017:4:Cd011998.

4. Kurella TM. Incidence, management, and outcomes of end-stage renal disease in the elderly. Curr Opin Nephrol Hypertens. 2009;18(3):252-7.

5. Bansal N. Evolution of cardiovascular disease during the transition to endstage renal disease. Semin Nephrol. 2017;37(2):120-31.

6. Wright J, Hutchison A. Cardiovascular disease in patients with chronic kidney disease. Vasc Health Risk Manag. 2009;5:713-22.

7. Nangaku M. Hypoxia and tubulointerstitial injury: a final common pathway to end-stage renal failure. Nephron Exp Nephrol. 2004;98(1):e8-12.

8. Hodgkins KS, Schnaper HW. Tubulointerstitial injury and the progression of chronic kidney disease. Pediatric Nephrology (Berlin, Germany). 2012;27(6): 901-9.

9. Lehnhardt A, Kemper MJ. Pathogenesis, diagnosis and management of hyperkalemia. Pediatric Nephrology (Berlin, Germany). 2011;26(3):377-84.

10. Kovesdy CP. Updates in hyperkalemia: outcomes and therapeutic strategies. Rev Endocr Metab Disord. 2017;18(1):41-7.
11. Chen W, Abramowitz MK. Metabolic acidosis and the progression of chronic kidney disease. BMC Nephrol. 2014;15:55.

12. Duranton F, Cohen G, De Smet R, Rodriguez M, Jankowski J, Vanholder R, et al. Normal and pathologic concentrations of uremic toxins. J Am Soc Nephrology. 2012;23(7):1258-70.

13. Deltombe O, Van Biesen W, Glorieux G, Massy Z, Dhondt A, Eloot S. Exploring protein binding of uremic toxins in patients with different stages of chronic kidney disease and during hemodialysis. Toxins. 2015;7(10):393346.

14. Mair RD, Sirich TL, Meyer TW. Uremic Toxin Clearance and Cardiovascular Toxicities. Toxins. 2018;10:6.

15. Stockler-Pinto MB, Saldanha JF, Yi D, Mafra D, Fouque D, Soulage CO. The uremic toxin indoxyl sulfate exacerbates reactive oxygen species production and inflammation in 3T3-L1 adipose cells. Free Radic Res. 2016;50(3):337-44.

16. Dias GF, Bonan NB, Steiner TM, Tozoni SS, Rodrigues S, Nakao LS, et al. Indoxyl Sulfate, a Uremic Toxin, Stimulates Reactive Oxygen Species Production and Erythrocyte Cell Death Supposedly by an Organic Anion Transporter 2 (OAT2) and NADPH Oxidase Activity-Dependent Pathways. Toxins. 2018;10:7.

17. Souma T, Abe M, Moriguchi T, Takai J, Yanagisawa-Miyazawa N, Shibata E, et al. Luminal alkalinization attenuates proteinuria-induced oxidative damage in proximal tubular cells. JASN. 2011;22(4):635-48.

18. Mahajan A, Simoni J, Sheather SJ, Broglio KR, Rajab MH, Wesson DE. Daily oral sodium bicarbonate preserves glomerular filtration rate by slowing its decline in early hypertensive nephropathy. Kidney Int. 2010;78(3):303-9.

19. Phisitkul S, Khanna A, Simoni J, Broglio K, Sheather S, Rajab MH, et al. Amelioration of metabolic acidosis in patients with low GFR reduced kidney endothelin production and kidney injury, and better preserved GFR. Kidney Int. 2010;77(7):617-23.

20. de Brito-Ashurst I, Varagunam M, Raftery MJ, Yaqoob MM. Bicarbonate supplementation slows progression of CKD and improves nutritional status. JASN. 2009;20(9):2075-84.

21. Goraya N, Simoni J, Jo C, Wesson DE. Dietary acid reduction with fruits and vegetables or bicarbonate attenuates kidney injury in patients with a moderately reduced glomerular filtration rate due to hypertensive nephropathy. Kidney Int. 2012;81(1):86-93.

22. Kim J, Shin W. How to do random allocation (randomization). Clin Orthopedic Surg. 2014;6(1):103-9.

23. Dettori J. The random allocation process: two things you need to know. Evidence-Based Spine-Care J. 2010;1(3):7-9.

24. Turner-Bowker DM, Bayliss MS, Ware JE Jr, Kosinski M. Usefulness of the SF-8 health survey for comparing the impact of migraine and other conditions. Qual Life Res. 2003;12(8):1003-12.

\section{Publisher's Note}

Springer Nature remains neutral with regard to jurisdictional claims in published maps and institutional affiliations.

Ready to submit your research? Choose BMC and benefit from:

- fast, convenient online submission

- thorough peer review by experienced researchers in your field

- rapid publication on acceptance

- support for research data, including large and complex data types

- gold Open Access which fosters wider collaboration and increased citations

- maximum visibility for your research: over $100 \mathrm{M}$ website views per year

At BMC, research is always in progress.

Learn more biomedcentral.com/submissions 